\title{
SNP rs657152 Is Not Associated with the Level of Viral Load in COVID-19 or the Probability of Disease in the Population of Caucasians in Eastern Siberia
}

\author{
E. A. Orlova ${ }^{a}$, O. B. Ogarkov ${ }^{a}, *$, P. A. Khromova ${ }^{a}$, V. V. Sinkov ${ }^{a}$, M. A. Khasnatinov ${ }^{a}$, \\ S. N. Zhdanova ${ }^{a}$, L. V. Rychkova ${ }^{a}$, and L. I. Kolesnikova ${ }^{a}$ \\ ${ }^{a}$ Scientific Center for Family Health and Human Reproduction Problems, Irkutsk, 664003 Russia \\ *e-mail: obogarkov@sbamsr.irk.ru
}

Received September 19, 2020; revised October 21, 2020; accepted October 27, 2020

\begin{abstract}
Cross-replicating associations with rs657152 at the 9q34.2c locus and rs11385942 at the $3 \mathrm{p} 21.31$ locus found in patients with severe COVID-19 in the Caucasian population require the study of the discovered phenomenon in various populations, including as an independent biological marker. Primers and TaqMan probes for PCR discrimination of the A and $\mathrm{C}$ alleles in single nucleotide polymorphism (SNP) rs657152 have been developed. The polymorphism of the rs657152 A/C locus was determined in 129 patients with COVID-19 and in a control group of 466 healthy individuals. There were no significant differences in the frequency of distribution of the $\mathrm{A}$ and $\mathrm{C}$ alleles, $0.47 / 0.53$ and $0.45 / 0.55$, between patients and healthy subjects, respectively. Also, no differences were found in the distribution of alleles in patients with a high viral load in the smear ( $\mathrm{Ct}$ in the range of 16-25) in comparison with an average and low viral load ( $\mathrm{Ct}$ in the range of 26-40).
\end{abstract}

Keywords: COVID-19, rs657152, allele discrimination, PCR, TaqMan probes

DOI: $10.1134 / \mathrm{S} 1022795421080093$

Severe acute respiratory syndrome coronavirus (SARS-CoV-2) was first identified in Wuhan, China, at the end of 2019, and the disease, dubbed COVID19, quickly became a global pandemic [1]. There is significant variation in the course of the disease among patients infected with the SARS-CoV-2 virus. In the early stages of the pandemic, countries in Europe faced far more COVID-19 cases and deaths than East Asia and Russia. The mechanisms that determine why some populations have more severe cases of the disease and others do not are poorly understood. The pathogenesis of severe forms of COVID-19 and associated respiratory failure has not been adequately studied; however, higher mortality is associated with older age and males [2, 3], a number of cardiovascular diseases and arterial hypertension, obesity, and diabetes [4-6]. Genome-wide association study (GWAS) [7] identified potential genetic factors involved in the development of COVID-19 [8]. A study of 1980 patients from Italy and Spain revealed cross-replicating associations with the rs 11385942 variant at the $3 \mathrm{p} 21.31$ locus and with rs657152 at the 9q34.2 locus, which were significant at the genomewide level [8].

The purpose of this work is to develop a PCR test with TaqMan probes suitable for discrimination of alleles $A$ and $C$ of rs657152 and perform a comparative assessment of the distribution of genotypes in groups of patients with COVID-19 and healthy individualsrepresentatives of the Caucasian population (Slavs) of Irkutsk oblast.

Compliance with ethical standards. This study was approved by the Ethics Committee of the Scientific Center for Family Health and Human Reproduction Problems. Collection of clinical material (nasal/pharyngeal swabs) was carried out as part of routine antiepidemic procedures for examining the population of Irkutsk oblast for the presence of the virus SARS$\mathrm{CoV}-2$. Isolation of RNA/DNA from the samples was carried out with the RealBest extraction kit; PCR diagnostics was conducted with the RealBest RNA SARS-CoV-2 kit. The group of patients included persons with a diagnosis COVID-19 and positive PCR test for the RNA of the virus. The healthy control included contact persons who underwent examination and did not have a positive PCR result for the RNA of the virus or diagnoses of viral respiratory infections or COVID-19. Calculation of the structure and length of primers and probes for discrimination of alleles A and $\mathrm{C}$ of rs657152 (Table 1) was produced manually according to the reference nucleotide sequence of the locus $A B O$ (https://www.ncbi.nlm.nih.gov/snp/ rs657152). 
Table 1. List of used primers and probes

\begin{tabular}{l|l|c|c}
\hline Primers/Probes & \multicolumn{1}{|c|}{ Sequence $5{ }^{\prime} \rightarrow 3^{\prime}$} & Amplicon length, bp & Annealing, ${ }^{\circ} \mathrm{C}$ \\
\hline $657152 \mathrm{~F}$ & TCCTACGGGAGGCAGCAGT & & 56 \\
\hline $657152 \mathrm{R}$ & AATTTAGGACATGTAAAGTTCA & \multirow{2}{*}{103} & 56 \\
\hline $657152 \mathrm{~A}$ & {$[$ FAM]TCTCGAATAGCTTCTTGAAAC[A-LNA]G[BHQ1] } & & 66 \\
\hline $657152 \mathrm{C}$ & {$[$ R6G]CTCGAATAGCTTCTTGAAAC[C-LNA]G[BHQ1] } & & 66 \\
\hline
\end{tabular}

Table 2. Characteristics of the surveyed samples

\begin{tabular}{l|c|c}
\hline \multicolumn{1}{c|}{ Indicator } & Control & Patients with COVID-19 \\
\hline$N$ & $N=466$ & $N=129$ \\
Men, \% $(N)$ & $33.0(154)$ & $41.1(53)$ \\
Women, \% $(N)$ & $67.0(312)$ & $58.9(76)$ \\
Age, years & $41.0 \pm 16.1$ & $38.8 \pm 21.5$ \\
Genotype rs657152 AA & 101 & 34 \\
Genotype rs657152 AC & 215 & 54 \\
Genotype rs657152 CC & 150 & 41 \\
Allele A & $417(0.45)$ & $122(0.47)$ \\
Allele C & $515(0.55)$ & $136(0.53)$ \\
Deviation from the Hardy-Weinberg equilibrium & $P>0.05$ & $P>0.05$ \\
\hline PCR threshold value (Ct) for detection SARS-CoV-2 & - & $35.0 \pm 5.5$ \\
\hline
\end{tabular}

The synthesis of primers and probes was carried out by Lumiprob RUS LLC. PCR was performed in the variant with real-time detection (RT-PCR) on a CFX amplifier (BioRad) using BioMaster HSqPCRHi-ROX (BIOLABMIX LLC) and equimolar concentrations of primers $(0.5 \mu \mathrm{M})$ and probes $(0.1 \mu \mathrm{M})$, respectively. Cycling regime: $95^{\circ} \mathrm{C}$ for $5 \mathrm{~min}$; 40 cycles, $95^{\circ} \mathrm{C}$ for $10 \mathrm{~s}, 55^{\circ} \mathrm{C}$ for $10 \mathrm{~s} ; 72^{\circ} \mathrm{C}$ for $10 \mathrm{~s}$. The calculation of statistical data, including the assessment of the deviation from the Hardy-Weinberg equilibrium, was carried out in Excel tables (Table 2); the deviations were statistically insignificant.

The initial objective of the study was to create two PCR tests to detect the cross-replicatingassociation rs657152 at the 9q34.2c locus and rs11385942 at the 3 p21.31 locus [8]. Unfortunately, detection of the $\mathrm{GA} / \mathrm{G}$ insertion-deletion at the $3 \mathrm{p} 21.31$ locus (rs11385942) by the TaqMan PCR test turned out to be technically difficult (data not shown), and the discrimination of alleles in the second test was not achieved. As a result, the study was carried out only for SNP rs657152.

The groups of patients and healthy controls did not differ significantly in sex and age. As one can see from Table 2, the volume of the obtained samples made it possible to achieve a ratio of genotypes that did not differ significantly from that expected according to Hardy-Weinberg. Analysis of the results obtained did not reveal any differences in the distribution of genotypes or alleles between the group of patients with
COVID-19 and the group of healthy individuals. We also failed to find significant differences in the distribution of alleles in COVID-19 patients with a high viral load in the smear (Ct in the range of 16-25) compared with COVID-19 patients with an average and low viral load ( $\mathrm{Ct}$ in the range of 26-40).

Comparison of the obtained allele frequencies with the data of the dbSNP database (https://www.ncbi. nlm.nih.gov/snp/rs657152\#frequency_tab) revealed significant differences $\left(\chi^{2}=13.2, P<0.001\right)$ of the studied control group in the frequency distribution of the $\mathrm{A}$ and $\mathrm{C}$ alleles $(0.45 / 0.55)$ in relation to the global $(0.39 / 0.61)$ distribution. A similar comparison of the distribution of the $A$ and $C$ alleles $(0.45 / 0.55)$ with the Caucasian population $(0.37 / 0.63)$ also showed significant differences $\left(\chi^{2}=23.1, P<0.001\right)$. It should be noted that the A allele of rs657152 is characterized as the "COVID-19 risk allele" together with the GA variant of rs11385942 [8]. The results obtained on the distribution of the A allele of rs657152 are the closest (geographically and ethnically) to its distribution in Estonia (0.44) and Finland (0.47) [8]. The study of the distribution of the rs657152 alleles on the territory of Russia, most likely, has not yet been carried out.

Thus, the identification of allele $A$ in the analysis of SNP rs657152 in the population of the Caucasian inhabitants (Slavs) of Irkutsk oblast alone cannot be considered a risk factor for a more severe course of COVID-19. 


\section{ACKNOWLEDGMENTS}

This work was carried out as part of the research work of the Scientific Center for Family Health and Human Reproduction Problems (Irkutsk) using the equipment of the Center for the Development of Progressive Personalized Health Technologies.

\section{COMPLIANCE WITH ETHICAL STANDARDS}

Conflict of interest. The authors declare that they have no conflict of interest.

Statement of compliance with standards of research involving humans as subjects. All procedures performed in a study involving people comply with the ethical standards of the institutional and/or national committee for research ethics and the 1964 Helsinki Declaration and its subsequent changes or comparable ethical standards.

Informed voluntary consent was obtained from each of the participants enrolled in the study.

\section{REFERENCES}

1. Zhu, N., Zhang, D., Wang, W., et al., A novel coronavirus from patients with pneumonia in China, 2019, N. Engl. J. Med., 2020, vol. 382, pp. 727-733. https://doi.org/10.1056/NEJMoa2001017

2. Zhou, F., Yu, T., Du, R., et al., Clinical course and risk factors for mortality of adult inpatients with COVID-19 in Wuhan, China: a retrospective cohort study, Lancet,
2020, vol. 395, pp. 1054-1062. https://doi.org/10.1016/S0140-6736(20)30566-3

3. Li, X., Xu, S., Yu, M., et al., Risk factors for severity and mortality in adult COVID-19 inpatients in Wuhan, J. Allergy Clin. Immunol., 2020, vol. 146, no. 1, pp. $110-118$.

https://doi.org/10.1016/j.jaci.2020.04.006

4. Chen, R., Liang, W., Jiang, M., et al., Risk factors of fatal outcome in hospitalized subjects with coronavirus disease 2019 from a nationwide analysis in China, Chest, 2020, vol. 158, no. 1, pp. 97-105. https://doi.org/10.1016/j.chest.2020.04.010

5. Docherty, A.B., Harrison, E.M., Green, C.A., et al., Features of 20133 UK patients in hospital with Covid19 using the ISARIC WHO Clinical Characterisation Protocol: prospective observational cohort study, $B M J$, 2020, vol. 369, p. 1985. https://doi.org/10.1136/bmj.m1985

6. Richardson, S., Hirsch, J.S., Narasimhan, M., et al., Presenting characteristics, comorbidities, and outcomes among 5700 patients hospitalized with COVID19 in the New York city area, JAMA, 2020, vol. 323, no. 20, pp. 2052-2059.

https://doi.org/10.1001/jama.2020.6775

7. Kraft, P., Zeggini, E., and Ioannidis, J.P.A., Replication in genome-wide association studies, Stat. Sci., 2009, vol. 24 , no. 4 , pp. $561-573$. https://doi.org/10.1214/09-STS290

8. Ellinghaus, D., Degenhardt, F., Bujanda, L., et al., Genome wide association study of severe Covid-19 with respiratory failure, N. Engl. J. Med., 2020. https://doi.org/10.1056/NEJMoa2020283 